\title{
Sporting myths: the REAL role of lactate during exercise
}

\author{
T Mann (BSc (Med)(Hon) Exercise Science
}

MRC/UCT Research Unit for Exercise Science and Sports Medicine, Department of Human Biology, Faculty of Health Sciences, University of Cape Town

\begin{abstract}
Background. Lactate or, as it was customarily known, 'lactic acid' was one of the first molecules to attract the attention of early exercise scientists, mainly because blood lactate concentration could be measured and was shown to increase with increasing exercise intensity. This connection resulted in lactate being associated with numerous other events associated with high-intensity exercise including muscle cramps, fatigue, acidosis and post-exercise muscle soreness. Nobel prize-winning research by AV Hill and Otto Meyerhof provided a rational explanation linking lactate to anaerobiosis and acidosis, which resulted in this relationship being widely accepted as fact. It was only following isotopic tracer studies of George Brooks and others that the true role of lactate during rest and exercise was revealed.

Conclusions. Lactate is now acknowledged as an important intermediate of carbohydrate metabolism, taken up from the blood by tissues such as skeletal and cardiac muscle as a substrate for oxidation. Furthermore, lactate formation consumes a proton, thereby buffering against muscle acidosis. For this reason, lactate production forms an essential aid to endurance performance rather than a hindrance.
\end{abstract}

\section{What is lactic acid?}

Complaints of legs feeling tired towards the end of a hard run and sore, stiff muscles the day after a race or hard training session have for many years been blamed on the presence of

\section{CORRESPONDENCE:}

Theresa Mann

MRC/UCT Research Unit for Exercise Science and Sports Medicine

PO Box 115

Newlands

7725 Cape Town

Tel: (021) 650-4569

Fax: (021) 686-7530

E-mail: mnnthe005@uct.ac.za lactic acid in the muscles. Briefly, lactic acid, or lactate, is one of two possible products produced by alternative reactions in the glycolytic pathway. ${ }^{1}$ The other product, pyruvate, is produced predominantly in the slow-twitch muscle fibres, whereas the poor blood supply and lower mitochondrial content of the fast-twitch muscle fibres favours lactate production. ${ }^{1}$ At physiological $\mathrm{pH}$, lactic acid is present in its de-protonated form, lactate. Thus, lactate production, and the assumed concomitant release of a hydrogen ion, has long been accepted as the cause of exercise-induced metabolic acidosis. ${ }^{1,4}$ The increase in blood lactate that occurs during intense exercise formed one of the landmark discoveries of early exercise scientists. Unfortunately, the limited knowledge and resources of the time lead to this phenomenon being somewhat misinterpreted and the resulting 'red herrings' continue to hamper many exercise physiologists decades later.

\section{History}

Lactic acid was first discovered in a sample of sour milk by Swedish chemist Carl Wilhelm Scheele in $1780 .^{2}$ Although initially greeted with scepticism, the existence of lactic acid was confirmed by 1810 , having been isolated from sources including fresh milk, ox meat and blood. ${ }^{2}$ A strong connection between lactic acid production and fermentation reactions was duly observed and lactic acid was commonly used to acidify foods and beverages as a form of preservative. ${ }^{2}$

The link between lactic acid and exercise may be largely attributed to the pioneering work of Otto Meyerhof and Archibald V Hill, who, in the early 1920s, studied the breakdown of carbohydrates in skeletal muscle. Meyerhof was particularly interested in the glycolytic pathway and was the first scientist to detail several of the reactions that occurred. He observed that lactic acid was produced from glycolysis when oxygen was absent and it was this discovery that added anaerobiosis (the absence of oxygen) to the existing link between lactic acid, fermentation and acidosis. ${ }^{3,4}$

This association between lactic acid, acidosis and anaerobiosis became relevant to exercise when Hill was able to quantify the amount of energy transferred when glucose was converted to lactate. His findings led him to propose that the energy of this reaction was enough to power muscle contraction. $^{3,4}$ Furthermore, Hill speculated that lactate production was initiated (i) when oxygen supply to the muscle was inadequate or (ii) when the energy demands of exercise 
exceeded what could be supplied using oxygen-dependent metabolism. Either of these conditions would necessitate an increase in anaerobic metabolism. Thus there appeared to be both evidence and rational explanation for the relationship between anaerobiosis, lactic acid production and acidosis. The fact that Meyerhof and Hill went on to share a Nobel prize for their findings in 1922 lead to these explanations being accepted as fact for a long period to come.

\section{Enduring misconceptions}

The relationship between inadequate oxygen supply to the muscles and excessive lactic acid production was assumed to be irrefutable and, in the same way, an increase in blood lactate was blamed for other events associated with highintensity exercise. These included muscle cramps, fatigue, post-exercise muscle soreness and even the formation of scar tissue.

Scientists were able to further explain the lactate accumulation supposedly responsible for these adverse events by measuring blood lactate at various exercise intensities. They observed that blood lactate concentration only began to increase at a particular point, later described as the 'anaerobic threshold'. The anaerobic threshold, also known as the 'lactate threshold', referred to an exercise intensity at which blood lactate steadily increased above the stable concentration observed at lower workloads. This was taken to represent the point at which oxygen supply to the muscle could not meet the demand placed on aerobic metabolism, forcing a switch to anaerobic metabolism to sustain muscle contractions.

Furthermore, it was initially believed that lactate could not be easily removed from the muscle and that some lactate remained behind in the muscle after exercise, resulting in pain and stiffness within a few hours.

\section{Discovery of lactate shuttles}

The concept of the 'anaerobic threshold', although still popular today among certain coaches and exercise scientists, was disproved less than a decade after its inception by George Brooks and his colleagues at the University of California (see review paper ${ }^{5}$ ). Through the infusion of radio-labelled carbon isotopes, incorporated into special 'tracer' lactate molecules, Brooks was able to track the transport of lactate within the body and also how it was metabolised. His revolutionary finding was that skeletal muscle is a major site of lactate oxidation as well as of lactate production. ${ }^{5}$ Special membrane proteins termed monocarboxylate transporters allow lactate to be transferred from its primary site of production in the fast-twitch glycolytic muscle fibres to the slow-twitch oxidative muscle fibres. ${ }^{5}$ At this point, lactate can be reconverted to pyruvate and used as a substrate for aerobic respiration.

The movement of lactate from its site of production to a site where it could be oxidised was dubbed lactate 'shuttling' and it is estimated that $75-80 \%$ of lactate produced during exercise is oxidised using this mechanism. ${ }^{5}$ Lactate can be shuttled between fibres of the same muscle or released into the blood for uptake by tissues, including active and inactive muscle, the liver and the heart. ${ }^{5}$ Skeletal muscles may continually release lactate into the blood stream without any noticeable change in blood lactate concentration. This is because the lactate is removed from the blood by other tissues, including the heart, muscle and liver, at a similar rate. Lactate that is not oxidised directly may be converted to glucose or to glycogen. ${ }^{5}$ Thus, in contrast to its previous reputation as being a dead-end metabolic waste product with destructive consequences, lactate is in fact a valuable distributor of carbohydrate potential energy within and between muscles.

With the evidence that lactate is continuously removed from the blood as well as being added to it, it becomes apparent that blood lactate concentration is not a good indicator of how much lactate is being produced by the muscles. Currently there is no convincing evidence that muscles ever become 'anaerobic' during exercise. ${ }^{5}$ Moreover, lactate formation has been shown to occur at rest and throughout exercise but is masked by the fact that an increase in lactate release into the blood usually initiates an increase in lactate removal. ${ }^{5}$ It is only at the exercise intensity previously termed the 'anaerobic threshold' that lactate release into the blood exceeds the rate at which it can be taken up by other tissues, causing the blood concentration to increase. This mismatch between lactate release and uptake occurs as a result of the increased rate of carbohydrate metabolism required to power high-intensity exercise.

\section{Is lactate accumulation bad?}

The long-standing association between lactate and anaerobiosis in the muscles appears largely unfounded; nevertheless, allegations of acidosis and muscle soreness following lactate accumulation in the muscle continue to persist.

To dispense with the latter, even before the discovery of the lactate shuttling systems, scientists had determined that blood and muscle lactate concentrations return to resting levels within an hour of the exercise session. It is therefore highly unlikely for lactate to cause the pain and stiffness that develops only 12 - 24 hours after exercise. ${ }^{6}$ Interestingly, postexercise massage, which may alleviate muscle discomfort, has no effect on blood lactate concentration. There is some evidence that lactate may even be beneficial for muscle recovery by serving as a substrate for glycogen re-synthesis. ${ }^{4}$ Post-exercise muscle soreness is now largely accepted to be as a result of mechanical damage to the muscle and the associated inflammation. ${ }^{5}$

Disproving the link between lactate accumulation and acidosis is a somewhat more complex affair. Innumerable investigations have shown an inextricable link between increased lactate concentration and decreased blood and muscle $\mathrm{pH}^{7}$ - long believed to result from the dissociation of protons associated with lactic acid. Only recently was this relationship shown to be a correlation only and not a 
cause-and-effect. Rather, it is proposed that rapid turnover of ATP, and not increased lactate production, is ultimately responsible for the accumulation of protons at high exercise intensity. ${ }^{4}$ Ironically, lactate is believed to resist rather than precipitate a fall in $\mathrm{pH}$ as formation of lactate from muscle glycogen actually consumes two protons. ${ }^{4}$

So is lactate accumulation bad from a muscle function perspective? The evidence is that lactate accumulation is not indicative of anaerobiosis, nor is it the cause of acidosis or post-exercise muscle soreness. To the contrary, lactate is particularly important for (i) the regeneration of the electron carrier, $\mathrm{NAD}^{+}$(ii) as a primary precursor for gluconeogenesis and (iii) as distributor of carbohydrate potential energy. Thus it appears that lactate accumulation is not inherently bad, indeed, it may even be argued as something of an advantage.

\section{REFERENCES}

1. Brooks G, Fahey T, Baldwin K. Glycogenolysis and glycolysis in muscle: the cellular degradation of sugar and carbohydrate to pyruvate and lactate. In: Exercise Physiology: Human Bioenergetics and Its Applications, 4th ed New York: McGraw Hill, 2005: 59-93.

2. Holten C, Muller A, Rehbinder D. Lactic Acid: Property and Chemistry of Lactic Acid and Derivatives. Germany: Verlag Chemie, 1971.

3. Hill A, Long $\mathrm{C}$, Lupton $\mathrm{H}$. Muscular exercise, lactic acid and the supply and utilization of oxygen. Proc $R$ Soc Lond B Biol Sci 1924; 16: 84-137.

4. Robergs R, Ghiasvand F, Parker D. Biochemistry of exercise-induced metabolic acidosis. Am J Physiol Regul Integr Comp Physiol 2004; 287 : R502-R516.

5. Brooks G. Intra- and extra-cellular lactate shuttles. Med Sci Sports Exerc 2000; 32(4): 790-9.

6. Maclntyre D, Reid W, McKensie D. Delayed muscle soreness. The inflammatory response to muscle injury and its clinical implications. Sports Med 1995; 20:24-40

7. Sahlin K, Harris R, Nylind B, Hultman E. Lactate content and $\mathrm{pH}$ in muscle samples obtained after dynamic exercise. Pflugers Arch 1976; 367:143-9.

\section{Clinical Sports Medicine \\ Medical Management and Rehabilitation, Text \\ with CD-ROM}

ISBN 1416024433 / $9781416024439 \cdot 512$ Pages $\cdot 336$

Illustrations

Saunders · Published September 2006

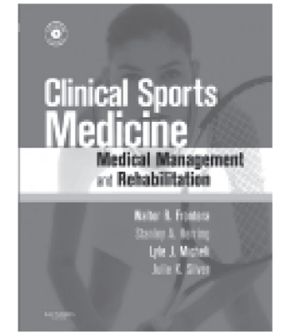

Only a fraction of sports medicine injuries require surgery, but most sports references emphasize surgical management over medical management and rehabilitation.

Clinical Sports Medicine: Medical Management and Rehabilitation is the only comprehensive sports medicine resource to focus on the medical management and rehabilitation of patients. Written by some of the most prominent names in Physical Medicine \& Rehabilitation, this book fills a void by providing an outstanding sports medicine reference aimed at non-surgeons. The international contributors to this book present you with global best practices from world leaders in the field. The main focus lies on the practical information you need to implement the most effective non-surgical management and rehabilitation approaches for today's diverse patient population. 